\title{
Effect of Sustained Isometric Handgrip Training on Blood Pressure in Normotensive and Prehypertensive Population
}

\author{
Parthkumar Devmurari ${ }^{1}$, Chandni Mehta ${ }^{2}$, Kalpesh Satani ${ }^{3}$, Lata Parmar ${ }^{4}$ \\ 1, 2, 3, 4 College of Physiotherapy, Sumandeep Vidyapeeth \\ Deemed to Be University, Piparia, Vadodara, Gujarat, India.
}

\section{ABSTRACT}

\section{BACKGROUND}

Prehypertension is common in adolescence and young adults and even normal blood pressure nowadays due to a marked increase in the sedentary lifestyle, job profile, psychological stress. Blood pressure (BP) in this age can linearly progress towards elevated levels of BP when associated with risk factors like body mass index (BMI). Along with lifestyle modifications, non-pharmacological interventions are also recommended to manage prehypertension and delay progression towards hypertension at a young age. Isometric handgrip training (IHGT) is emerging as a popular non-pharmacological therapy. Research has demonstrated hypotensive effects with IHGT in various sets when performed at 30 - $40 \%$ maximum voluntary contraction (MVC). The purpose of the present study was to find the effect of isometric handgrip strength training (IHG) on blood pressure and pulse pressure in normotensive and prehypertensive population.

\section{METHODS}

An experimental study design where 26 participants meeting the inclusion criteria were selected by convenient sampling. They were equally grouped into normotensive and prehypertensive training groups after measurement of baseline BP. All of them performed five 2 - minute sustained isometric contractions, at 30 - $40 \%$ of MVC, using 90 an inexpensive spring hand grip trainer, for six sessions per week for 2 weeks. MVC was determined by a hand dynamometer. Pre - to - post BP and PP were compared in the present study.

\section{RESULTS}

After 2 weeks, t-test and repeated measures of ANOVA revealed a significant reduction in systolic blood pressure (SBP) [Mean Difference 4, 9] and diastolic blood pressure (DBP) [Mean Difference $-5,5](\mathrm{P}=0.01)$ but PP did not change in normotensive $(\mathrm{P}-0.64)$ and prehypertensives $(\mathrm{P}=0.40)$.

\section{CONCLUSIONS}

Practicing IHGT using an inexpensive spring handgrip trainer for two weeks consecutively reduced blood pressure but not pulse pressure.

\section{KEY WORDS}

Isometric, Handgrip. Blood Pressure.
Corresponding Author: Dr. Parthkumar Devmurari, College of Physiotherapy, Sumandeep Vidyapeeth an Institution Deemed to Be University, Piparia, Waghodia Road, Waghodia, Vadodara, Gujarat - 391760, India.

E-mail: devmurariparth@gmail.com

DOI: $10.14260 /$ jemds/2021/636

How to Cite This Article:

Devmurari P, Mehta C, Satani K, et al. Effect of sustained isometric handgrip training on blood pressure in normotensive and prehypertensive population. J Evolution Med Dent Sci 2021;10(36):3121-3126, DOI: 10.14260/jemds/2021/636

Submission 28-04-2021,

Peer Review 13-06-2021,

Acceptance 19-06-2021,

Published 06-09-2021.

Copyright (C) 2021 Parthkumar Devmurari et al. This is an open access article distributed under Creative Commons Attribution License [Attribution 4.0 International (CC BY 4.0)] 


\section{BACKGROUND}

Blood pressure (BP) refers to the force the blood exerts against a vessel wall. It is measured in millimetres of mercury ( $\mathrm{mm}$ $\mathrm{Hg}$ ) and recorded in the form of a fraction (e.g., 119 / 79). The number given above states systolic whereas the one given below indicates diastolic pressure. The systolic pressure is the uppermost category of pressure expelled against the arterial wall in the body. The diastolic pressure (which is constantly present) is the lowest. Systolic and diastolic pressures have their own normative and specific ranges and elevation from a certain level determines the abnormal rise (Hypertension) in the blood pressure. ${ }^{1}$

Hypertension - HTN, is a major problem worldwide.2,3 Elderly population and adult population are more susceptible to get cardiac and vascular disorders due to persistently elevated blood pressure. ${ }^{4}$ It is considered as a powerful risk factor for cardiovascular disease, $2,5,6,7,8$ and is one of the most preventable causes of premature morbidity and mortality worldwide. ${ }^{9}$

Studies suggest that every 20 millimetres of $\mathrm{Hg}$ rise in pressure is subjected to be at risk of getting cardiovascular diseases (CVDs) with short duration (approximately 5 years) which is two-fold of normal in middle-aged and elderly.4 Prevalence of hypertension and incidence of CVD both rises with age advancement as both are associated with each other. In addition, data obtained from the Framingham Heart Study indicated systolic blood pressure between 130 - 139 and diastolic blood pressure between 80 - $85 \mathrm{mmHg}$ relatively rise almost two-fold risk in the prevalence of CVDs as compared to normotensive. ${ }^{2}$

The individuals with a high body mass index are more susceptible to hypertension from prehypertension. $6,8,10,11$ Several pathophysiological changes like increase in sympathetic activity, decrease in arterial elasticity and hyperinsulinemia associated with an increase in body mass and unhealthy lifestyle have been implicated in the pathogenesis of hypertension. ${ }^{12}$

Along with prehypertension, the increasing prevalence of obesity is also seen in early life. ${ }^{13}$ However, an increase in non -essential and essential fat storages in adipose tissues might have an association with vascular reduction in distensibility. Pulse Pressure - PP is a reliable indicator of vascular distensibility.12-15 Thus, it becomes important to keep the systolic and diastolic blood pressure within the normal range not only for middle-aged hypertensives but also for normotensive and / or prehypertensive adolescents and young adults. ${ }^{16}$

Antihypertensive drug's effectiveness is often inconsistent $^{6}$ and studies suggest that almost half of the hypertensive population receiving antihypertensive drug treatments may have increased levels of arterial pressure. ${ }^{7}$ The incidence of poor control over HTN is thought to be due to less adherence towards prescription. ${ }^{5}$ The need of availability and applicability of alternative therapies are indeed necessary for the treatment of hypertension. 5,7

For lowering the blood pressure, proven approaches like programs for weight loss, dietary changes, aerobic exercises, etc., are well known. Aerobic exercises such as walking, marching on a place and bicycling have been recommended for controlling / maintaining SBP and DBP as a result of which reduction of blood pressure in hypertensive population were found significant during the study. $3,17,18,19,20$
Dynamic exercises have been advocated as one of the aiding method programs for the treatment of HTN.21,22

Resistance training can also be performed as isometric or static which involves a change in tone without change in muscle length. ${ }^{3}$ Nowadays, isometric exercise alone is promoted as an approach to lower the resting blood pressure. ${ }^{23}$ Furthermore, there is a need for alternative therapy in the current scenario of the rise in HTN incidence worldwide. ${ }^{5,7}$ Isometric handgrip training (IHG) is becoming popular in recent times and the technique is revealing the effect of hypotension. $5,7,24,25,26,27$

Evidence suggest that isometric exercises are more beneficial in the view of less rise in systolic pressure in relation to the rise during various aerobic exercise protocols. 18,19,20

Studies suggest that normotensive and prehypertensive with resistance training have positive effects on systemic vascular pressures. No evidence was found which states an increase in the resting blood pressure due to resistance training in normotensive or prehypertensive subjects. 5,7,3,24,25,27,28

Evidence also suggests that IHGT can alter the PP when practised over an extended period. ${ }^{29}$ although isometric handgrip training might not be efficient to use as a sole treatment and can be used as an adjunct to the present pharmacological treatment; ${ }^{25}$ independent of medication. ${ }^{5}$ Handheld dynamometer is a standardized device to measure isometric strength. ${ }^{3,5}$ However; other cost-effective devices are also showing equal results.3,5,7 When compared to prescribed pharmacological treatment for antihypertensives, digital handgrip machines are cost-effective, however, for some patients this device may still increase the economic burden. ${ }^{7}$

Research suggests that aerobic exercise modalities involving more than 150 mins / week take a long time in normalizing blood pressure when compared to isometric exercises. ${ }^{3}$ It is evident that if regular resistance training (isometric resistance) is incorporated at the workplace then the incidence of hypertension can be brought down to a significant level also, resistance training (isometric) might be useful in dealing with HTN patients or for the preventive purpose when incorporated with pharmacological intervention. $4,24,26$ In the present study, the isometric handgrip training was given with an inexpensive spring handgrip trainer. As new training programmes are needed to be evaluated for producing the BP-lowering effect, the present study had a set protocol of five 2 - minute's contractions with intermittent 1 - minute rest periods. Total six consecutive sessions were performed within a week for two weeks.

\section{Objectives}

1. To see the effect of IHG training on blood pressure (BP) and pulse pressure (PP) in normotensives

2. To see the effect of IHG training on blood pressure (BP) and pulse pressure (PP) in Prehypertensives.

\section{METHODS}

The experimental study was approved by the Sumandeep Vidyapeeth Institutional ethics committee, and was conducted by the Physiotherapy department (College of physiotherapy) from August 2013 to July 2014 
Normotensive and Prehypertensive ${ }^{2}$ students from Sumandeep Vidyapeeth, Piparia were selected as subjects. A total of 30 males and females were recruited based on their blood pressures with who were healthy and not having any known cardio-pulmonary or any musculoskeletal condition hampering grip strength. Classification of blood pressure by JNC. ${ }^{2}$

\begin{tabular}{|ccc|}
\hline $\begin{array}{c}\text { Blood Pressure } \\
\text { Classification }\end{array}$ & SBP $(\mathbf{m m H g})$ & DBP $(\mathbf{m m H g})$ \\
\hline Normal & $<120$ & $<80$ \\
Prehypertensive & $120-139$ & $80-89$ \\
\hline \multicolumn{3}{c}{ Table } \\
\hline
\end{tabular}

\section{Procedure}

Handgrip strength was assessed with JAMAR hydraulic hand dynamometer. ${ }^{30}$ (calibrated) This measured participants' maximum voluntary contraction (MVC). Based on their MVC, they were taught to perform sustained isometric contractions at approx. $30 \%-40 \%$ of MVC using spring, systolic and diastolic blood pressure (SBP and DBP), pulse pressure (PP), grip strength (kg).

\section{Statistical Analysis}

It was done using SPSS software, version 14. Comparison of blood pressure reduction within normotensive and prehypertensive groups at different time intervals were done using repeated measures of ANOVA. Two samples independent t-test was used to check the difference in DBP \& SBP at different time intervals between the two groups.

The difference in the grip strength and pulse pressure, before and after the training within the prehypertensive group was done using paired t-test. All statistical analyses were carried out at a $95 \%$ confidence level with statistical significance (P-value) at less than 0.05 .

\section{RESULTS}

\begin{tabular}{|ccc|}
\hline Variables & $\begin{array}{c}\text { Normotensives N = 13 } \\
\text { Mean (SD) }\end{array}$ & $\begin{array}{c}\text { Prehypertensives N = } \\
\text { 13 Mean (SD) }\end{array}$ \\
Age (Years) & $20.5(2.15)$ & $20.1(2.18)$ \\
BMI (Kg / m $\left.{ }^{2}\right)$ & $21.46(3.2)$ & $25.1(5.1)$ \\
Baseline SBP (mmHg) & $115(4.75)$ & $129.5(6.5)$ \\
Baseline DBP (mmHg) & $70(2.72)$ & 79.38 \\
Pulse Pressure & $47.1(7.0)$ & 48.4 \\
Grip strength Right & $19(7.5)$ & $23(8.3)$ \\
Grip strength Left & $18.9(7.2)$ & $22.8(9.7)$ \\
\hline \multicolumn{2}{|c}{ Table 1. Demographic Variables } \\
\hline
\end{tabular}

\begin{tabular}{|cccc|}
\hline Blood Pressure & Hotelling's Trace & F Value & P - Value \\
SBP & 2.10 & 6.6 & 0.01 \\
DBP & 1.15 & 3.6 & 0.01 \\
\hline Table 2. Comparison of Reduction in Blood Pressure $(\mathbf{m m H g})$ at \\
Different Time Interval within Normotensive and Prehypertensive \\
Groups Using Repeated Measures of ANOVA
\end{tabular}

\begin{tabular}{|cccc|}
\hline Readings of SBP & $\begin{array}{c}\text { NormotensivesPrehypertensives } \\
\text { Mean (SD) }\end{array}$ & Pean (SD) & - Value \\
Reading - 1 & $4.7(2.4)$ & $6.2(2.19)$ & 0.08 \\
Reading - 2 & $4.15(2.37)$ & $6.5(1.45)$ & 0.004 \\
Reading - 3 & $6.3(3.9)$ & $7.8(2.79)$ & 0.243 \\
Reading - 4 & $5.2(4.2)$ & $7.8(5.57)$ & 0.182 \\
Reading - 5 & $4.9(2.78)$ & $6(4.22)$ & 0.445 \\
Reading - 6 & $4.9(1.55)$ & $5.4(2.44)$ & 0.526 \\
\hline Table 3. A Difference of the Difference in SBP at Different Time \\
Intervals between the Two Groups Using Two Sample Independent t - \\
test \\
\hline \multicolumn{4}{c}{} \\
\hline
\end{tabular}

\begin{tabular}{|cccc|}
\hline $\begin{array}{c}\text { Readings of } \\
\text { DBP }\end{array}$ & $\begin{array}{c}\text { Normotensives Mean } \\
\text { (SD) }\end{array}$ & $\begin{array}{c}\text { Prehypertensives Mean } \\
\text { (SD) }\end{array}$ & $\begin{array}{c}\text { P - } \\
\text { Value }\end{array}$ \\
Reading - 1 & $4.1(4.3)$ & $4.4(2.62)$ & 0.845 \\
Reading - 2 & $4.7(3.87)$ & $6(3.23)$ & 0.378 \\
Reading - 3 & $5.3(2.63)$ & $6.5(3.54)$ & 0.336 \\
Reading - 4 & $5.3(2.87)$ & $7.2(3.72)$ & 0.153 \\
Reading - 5 & $3.5(5.1)$ & $5(4.05)$ & 0.416 \\
Reading - 6 & $5.5(1.8)$ & $4.7(3.09)$ & 0.415 \\
\hline Table 3. B Difference of the Difference in DBP at Different Time \\
Intervals between the Two Groups Using Two Sample Independent t- \\
\multicolumn{4}{|c|}{ Test } \\
\hline
\end{tabular}

\begin{tabular}{|cccc|}
\hline $\begin{array}{c}\text { Pulse Pressure } \\
\text { (mmHg) }\end{array}$ & $\begin{array}{c}\text { Before Mean } \\
\text { (SD) }\end{array}$ & After Mean (SD) & P - Value \\
Normotensive group & $47.1(7.0)$ & $47.6(7.0)$ & 0.64 \\
$\begin{array}{c}\text { Prehypertensive } \\
\text { group }\end{array}$ & $48.4(6.9)$ & $44.4(15.7)$ & 0.40 \\
\hline $\begin{array}{c}\text { Table 4. Comparison of Change in Pulse pressure (mmHg) in } \\
\text { Normotensive and Prehypertensive Groups Using Paired } \text {-Test }\end{array}$ \\
\hline
\end{tabular}

\begin{tabular}{|cccc|}
\hline $\begin{array}{c}\text { Grip Strength } \\
\text { (kg) }\end{array}$ & $\begin{array}{c}\text { Before Mean } \\
\text { (SD) }\end{array}$ & After Mean (SD) & P - Value \\
Right hand & $21.0(7.8)$ & $24.0(8.5)$ & 0.00 \\
Left hand & $20.6(8.1)$ & $23.9(8.7)$ & 0.00 \\
\hline Table 5. Comparison of Grip - Strength before and after Training in \\
Prehypertensive Group Using Paired -Test \\
\hline
\end{tabular}

\section{DISCUSSION}

The participants were allotted according to their level of blood pressure into their respective groups. There were 15 subjects in each group at the beginning of the study, but later on, 2 subjects (both males) from the normotensive group, 2 subjects (01 male and 01 female) from the prehypertensive group had to be excluded as they did not report for baseline blood pressure measurements after the completion of treatment. The age - range of those included was 17 - 25 years.

In the present study, both the groups performed isometric contractions using an inexpensive handgrip trainer. Several studies have been done with varying training protocols for isometric handgrip training - IHGT having a frequency of 3 - 5 days per week for 4 - 10 weeks, with 30 - $40 \%$ MVC. 5,7,23,24,25,26,27,31,32 The results of all the studies showed a significant reduction in SBP and DBP, either one or both.

The present study evaluated the results of exercise protocol, 6 sessions per week for two weeks. During each session, participants from both groups performed five 2 - minutes contractions with 30 - $40 \%$ MVC with alternate hands using a spring handgrip trainer. A resting period of 2 - minutes was given between each contraction. The chosen protocol in the current study showed a significant reduction in SBP and DBP for both the groups' post-training $(\mathrm{P}=0.01)$.

The low normotensive range although, is known to linearly predict progression to hypertension in young adulthood as suggested by Amir Tirosh and colleagues (2010), studied the role of body composition on gender and other parameters to determine the likelihood of having a risk of hypertension in adults. 6

The mean BMI in the present study for the prehypertensive group $\left(25.1 \mathrm{~kg} / \mathrm{m}^{2}\right)$ was significantly higher than that of the normotensive group. Report from WHO states that for the Asian population, BMI values of $23 \mathrm{~kg} / \mathrm{m}^{2}$ or higher, represent increased risk. Li Col SP Singh et al. (2008) suggested lower BMI cut-offs for overweight and obesity in Indian populations. ${ }^{33}$ Empar Lurbe et al. (2001) ${ }^{13}$ reports that obesity is positively related to systolic BP even in early life. 
Bonita Falkner et al. (2007) ${ }^{32}$ stated that blood pressure is influenced significantly by initial BMI changes.

In the current study, levels of PA of the participants, as low, moderate and high were determined using the 'last 7 - day', self -administered version International Physical Activity Questionnaire - IPAQ. Hendrik JF Helmerhorst et al. (2012) ${ }^{34}$ reviewed the efficacy of physical activity questionnaires in terms of its reliability and validity which stated that IPAQ is used commonly in many countries and shows good results. Carlos A Celis - Morales et al. (2012) ${ }^{35}$ indicated that the IPAQ with more accuracy than physical activity. Sedentary lifestyle (behaviour) was more definite in relation with increased cardiometabolic risk profile whereas, there was no association to physical activity (Moderate to vigorous) and. ${ }^{35}$

The relationship between three different levels of PA and blood pressure revealed no significant association in the present study and this probably could be attributed to the small sample size. However, Augusto Ce'sar Ferreira de Moraes et al. (2013) ${ }^{36}$ reported that there is a direct relation in adolescent of physical activity (AP) and sedentary behaviour (SB) with blood pressure and increased SBP levels in boys found in which less than recommended levels of Physical Activity from 2 hours ranging to 4 hours per day. ${ }^{36}$

Chester A. Ray and Dario I. Carrasco (2000) illustrated that after 5 weeks of IHGT, there was a reduction in diastolic arterial pressure (5 $\mathrm{mmHg}$ ), while negligible changes were seen in SBP among normotensive subjects (19 - 35 years) at rest. ${ }^{4}$ However, Ve'ronique A. Cornelissen et al. (2011) ${ }^{29}$ and Mark B. Badrov (2013) ${ }^{37}$ showed that instead of a reduction in both variables of BP, reductions in resting SBP were somewhat more pronounced after IHGT of minimum 6 weeks ${ }^{29}$ and 8 weeks respectively. ${ }^{37}$

The normotensive group in the present study, at the end of two weeks, showed a reduction in SBP. It is suggested that minute changes in SBP and DBP might be because of the normotensive population in some studies. ${ }^{5,24}$ The prehypertensive group illustrated a significant reduction of SBP and DBP in the present study. Philip J. Millar and colleagues (2007) ${ }^{27}$ repeated the same protocol, as they had used in their study on normotensive subjects, ${ }^{27}$ and reported a reduction in SBP and DBP in medicated hypertensive person ${ }^{28}$ whose results were found similar to the present study. Peters et al. $(2006)^{38}$ studied the IHG training protocol on hypertensive individuals for 6 weeks and the outcome was an effective reduction in SBP $(\approx 13 \mathrm{mmHg})$ but not in diastolic blood pressure in prehypertensive and hypertensive individuals..$^{5,7}$

In the present study apart from pre and post-baseline measurements, readings of blood pressure were taken during two weeks of the training period. The mean values are shown in Tables - 2 and 3 with the $\mathrm{P}$ - values. The immediate pre-post values of SBP and DBP had shown some changes; however, they were statistically insignificant.

Tables -3 and 4 show the magnitudes of reduction in SBP and DBP immediately after a single training session. There was a definite change between the first reading and the last reading for SBP and DBP, showing some reduction in both the groups. For the last reading, the SBP values were closer for both groups (Table - 3), than they were for reading - 1.

As per the JNC statement (2003), hypertension of diastolic pressure prevails early age of above 50 years, is more at risk of cardiovascular condition when alone rise in pressure or combination with SBP. Thus, diastolic blood pressure is more quantified sedentary behaviour parameters were measured

significant than systolic blood pressure and it should be treated accordingly. ${ }^{13}$

The present study is in agreement with a number of studies that showed a reduction in BP of the prehypertensive group than the normotensive group, thus indicating beneficial effects of simple IGHT. In the present study, the values of PP changed but are insignificant at the end of two weeks. Cheri $\mathrm{L}$. McGowan et al. who showed that 8 weeks of IHG training did not alter the endothelium-dependent vasodilatory response. ${ }^{39,40}$ Andrea C. Taylor et al. (2002), showed changes in the sympathovagal balance following 10 weeks' training. ${ }^{37,41}$ The grip strength was also assessed at the end of two weeks in the present study, which significantly increased for all the participants with the training. Steven G. Chrysant (2010) said that besides blood pressure, isometric exercise is also associated with other beneficial effects consisting of an increase in upper body strength. ${ }^{21}$

\section{CONCLUSIONS}

The positive effect of IGHT was found on SBP and DBP in normotensives and prehypertensives. There was no alteration in pulse pressure for either normotensives or prehypertensives after 2 weeks of isometric handgrip training. There was a significant association between BMI and blood pressure in persons with prehypertension. There was no significant association between levels of physical activity and blood pressure for normotensives and prehypertensives in the present study. The grip strength was significantly increased after 2 weeks of training for all the participants.

Data sharing statement provided by the authors is available with the full text of this article at jemds.com.

Financial or other competing interests: None.

Disclosure forms provided by the authors are available with the full text of this article at jemds.com.

\section{REFERENCES}

[1] O'Sullivan SB, Schmitz TJ, Fulk G. Physical rehabilitation. $7^{\text {th }}$ edn. FA Davis Company, 2019: p. 50-69.

[2] Chobanian AV, Bakris GL, Black HR, et al. Seventh report of the joint national committee on prevention, detection, evaluation and treatment of high blood pressure. Hypertension 2003;42(6):1206-52.

[3] Brook RD, Appel LJ, Rubenfire M, et al. Breyond medications and diet: alternative approaches to lowering blood pressure: a scientific statement from the American Heart Association. Hypertension 2013;61(6):1360-83.

[4] Pletcher MJ, Bibbins-Domingo K, Lewis CE, et al. Prehypertension during young adulthood and coronary calcium later in life. Annals of Internal Medicine 2008;149(2):91-9.

[5] Millar PJ, Paashuis A, McCartney N. Isometric handgrip effects on hypertension. Current Hypertension Reviews 2009;5(1):54-60.

[6] Tirosh A, Afek A, Rudich A, et al. Progression of normotensive adolescents to hypertensive adults: a study of 26980 teenagers. Hypertension 2010;56(2):203-9.

[7] Millar PJ, Bray SR, MacDonald MJ, et al. The hypotensive effects of isometric handgrip training using an J Evolution Med Dent Sci / eISSN - 2278-4802, pISSN - 2278-4748 / Vol. 10 / Issue 36 / Sept. 06, 2021 
inexpensive spring handgrip training device. Journal of Cardiopulmonary Rehabilitation and Prevention 2008;28(3):203-7.

[8] Falkner B. Hypertension in children and adolescents: epidemiology and natural history. Pediatric Nephrology 2010;25(7):1219-24.

[9] Williams B, Williams H, Northedge J, et al. Hypertension: the clinical management of primary hypertension in adults. Update of Clinical Guidelines 18 and 34. NICE clinical No. 127. 2011. (Accessed August 2011). http://guidance.nice.org.uk/cg127.

[10] Torrance B, McGuire KA, Lewanczuk R, et al. Overweight, physical activity and high blood pressure in children: a review of the literature. Vascular Health And Risk Management 2007;3(1):139.

[11] Who Expert Consultation. Appropriate body-mass-index for Asian populations and its implications for policy and intervention strategies. Lancet (London, England) 2004;363(9403):157-63.

[12] Shaikh WA, Patel M, Singh SK. Association of adiposity with pulse pressure amongst Gujarati Indian adolescents. Indian Journal of Community Medicine 2010;35(3):406-8.

[13] Lurbe E, Alvarez V, Redon J. Obesity, body fat distribution, and ambulatory blood pressure in children and adolescents. The Journal of Clinical Hypertension. 2001;3(6):362-7.

[14] Drukteinis JS, Roman MJ, Fabsitz RR, et al. Cardiac and systemic hemodynamic characteristics of hypertension and prehypertension in adolescents and young adults: the Strong Heart Study. Circulation 2007;115(2):221-7.

[15] Benetos A, Rudnichi A, Safar M, et al. Pulse pressure and cardiovascular mortality in normotensive and hypertensive subjects. Hypertension 1998;32(3):560-4.

[16] Falkner B, Gidding SS, Portman R, et al. Blood pressure variability and classification of prehypertension and hypertension in adolescence. Pediatrics 2008;122(2):238-42.

[17] Mir F, Brown M, O'Shaughnessy K, et al. Guidelines for the Management of Hypertension. Clinical Pharmacology Unit, Cambridge University Hospitals Guidelines, 2010.

[18] Schjerve IE, Tyldum GA, Tjønna AE, et al. Both aerobic endurance and strength training programmes improve cardiovascular health in obese adults. Clinical Science (Lond) 2008;115(9):283-93.

[19] Collier SR, Kanaley JA, Carhart R, et al. Effect of 4 weeks of aerobic or resistance exercise training on arterial stiffness, blood flow and blood pressure in pre - and stage1 hypertensives. Journal of Human Hypertension 2008;22(10):678-86.

[20] Cardoso CG Jr, Gomides RS, Queiroz ACC, et al. Acute and chronic effects of aerobic and resistance exercise on ambulatory blood pressure. Clinics 2010;65(3):317-25.

[21] Chrysant SG. Current evidence on the hemodynamic and blood pressure effects of isometric exercise in normotensive and hypertensive persons. The Journal of Clinical Hypertension 2010;12(9):721-6.

[22] Kelley GA, Kelley KS. Progressive resistance exercise and resting blood pressure: a meta - analysis of randomized controlled trials. Hypertension 2000;35(3):838-43.

[23] Kelley GA, Kelley KS. Isometric handgrip exercise and resting blood pressure: a meta-analysis of randomized controlled trials. Journal of Hypertension 2010;28(3):411-8.
[24] Ray CA, Carrasco DI. Isometric handgrip training reduces arterial pressure at rest without changes in sympathetic nerve activity. American Journal of Physiology - Heart and Circulatory Physiology 2000;279(1):H245-9.

[25] Taylor AC, McCartney NE, Kamath MV, et al. Isometric training lowers resting blood pressure and modulates autonomic control. Medicine and Science in Sports and Exercise 2003;35(2):251-6.

[26] Wiley RL, Dunn CL, Cox RH, et al. Isometric exercise training lowers resting blood pressure. Medicine and Science in Sports and Exercise 1992;24(7):749-54.

[27] Millar PJ, Bray SR, McGowan CL, et al. Effects of isometric handgrip training among people medicated for hypertension: a multilevel analysis. Blood Pressure Monitoring 2007;12(5):307-14.

[28] Millar PJ, Swaine IL, McGowan CL. Impact of resistance training on blood pressure: are all contractions created equal? Hypertension 2012;59(4):e35.

[29] Cornelissen VA, Fagard RH, Coeckelberghs E, et al. Impact of resistance training on blood pressure and other cardiovascular risk factors: a meta-analysis of randomized, controlled trials. Hypertension 2011;58(5):950-8.

[30] Hamilton GF, McDonald C, Chenier TC. Measurement of grip strength: validity and reliability of the sphygmomanometer and jamar grip dynamometer. Journal of Orthopaedic \& Sports Physical Therapy 1992;16(5):215-9.

[31] John EB, Popoola AO, Allen AA. Eight-days isometric exercise training lowers blood pressure in normotensive subjects. J Clinical Hypertension 2008;10(5(Suppl A): A59.

[32] McGowan CL, Levy AS, McCartney N, et al. Isometric handgrip training does not improve flow-mediated dilation in subjects with normal blood pressure. Clinical Science 2007;112(7):403-9.

[33] Singh SP, Sikri G, Garg MK. Body mass index and obesity: tailoring "cut - off" for an Asian Indian male population. Medical Journal Armed Forces India 2008;64(4):350-3.

[34] Helmerhorst HJF, Brage S, Warren J, et al. A systematic review of reliability and objective criterion-related validity of physical activity questionnaires. International Journal of Behavioral Nutrition and Physical Activity 2012;9(1):103.

[35] Celis-Morales CA, Perez-Bravo F, Ibañez L, et al. Selfreported physical activity and sedentary time: effects of measurement method on relationships with risk biomarkers. PLoS One 2012;7(5):e36345.

[36] de Moraes AC, Carvalho HB, Rey-López JP, et al. Independent and combined effects of physical activity and sedentary behavior on blood pressure in adolescents: gender differences in two cross-sectional studies. PLoS One 2013;8(5):e62006.

[37] Badrov MB, Bartol CL, DiBartolomeo MA, et al. Effects of isometric handgrip training dose on resting blood pressure and resistance vessel endothelial function in normotensive women. European Journal of Applied Physiology 2013;113(8):2091-100.

[38] Peters PG, Alessio HM, Hagerman AE, et al. Short-term isometric exercise reduces systolic blood pressure in hypertensive adults: possible role of reactive oxygen species. International Journal of Cardiology 2006;110(2):199-205. 
[39] McGowan CL, Levy AS, Millar PJ, et al. Acute vascular responses to isometric handgrip exercise and effects of training in persons medicated for hypertension. American Journal of Physiology - Heart and Circulatory Physiology 2006;291(4):H1797-802.

[40] McGowan CL, Levy AS, McCartney N, et al. Isometric handgrip training does not improve flow-mediated dilation in subjects with normal blood pressure. Clinical Science 2007;112(7):403-9.

[41] Frese EM, Fick A, Sadowsky HS. Blood pressure measurement guidelines for physical therapists. Cardiopulmonary Physical Therapy Journal 2011;22(2):5-12 